\title{
Particle diffusion in complex nanoscale pore networks
}

Müter, Dirk; Sørensen, Henning Osholm; Bock, H.; Stipp, Susan Louise Svane

Published in:

Journal of Physical Chemistry C

DOI:

10.1021/jp5117867

Publication date:

2015

Document version

Peer reviewed version

Document license:

Other

Citation for published version (APA):

Müter, D., Sørensen, H. O., Bock, H., \& Stipp, S. L. S. (2015). Particle diffusion in complex nanoscale pore networks. Journal of Physical Chemistry C, 119(19), 10329-10335. https://doi.org/10.1021/jp5117867 


\title{
Particle diffusion in complex nanoscale pore
}

\section{networks}

D. Müter ${ }^{1, *}$, H.O. Sørensen ${ }^{l}$, H. Bock ${ }^{2}$, S.L.S. Stipp ${ }^{1}$

${ }^{1}$ Nano-Science Center, Department of Chemistry, University of Copenhagen, Copenhagen, Denmark

${ }^{2}$ Department of Chemical Engineering, Heriot-Watt University, Edinburgh, Scotland, United Kingdom

KEYWORDS: X-Ray Tomography; DPD Simulations; Diffusion; Tortuosity; Chalk; Adsorption

\begin{abstract}
We studied the diffusion of particles in the highly irregular pore networks of chalk, a very fine grained rock, by combining three dimensional X-ray imaging and dissipative particle dynamics (DPD) simulations. X-ray imaging data were collected at $25 \mathrm{~nm}$ voxel dimension for two chalk samples with very different porosities (5\% and $26 \%$ ). The three dimensional pore systems derived from the tomograms were imported into DPD simulations and filled with spherical particles of variable diameter and with an optional attractive interaction to the pore surfaces. We found that diffusion significantly decreased to as much as $60 \%$ when particle size increased from $1 \%$ to $35 \%$ of the average pore diameter). When particles were attracted to the pore surfaces,
\end{abstract}


even very small particles, diffusion was drastically inhibited, by as much as a factor of 100 . Thus, the size of particles and their interaction with the pore surface strongly influence particle mobility and must be taken into account for predicting permeability in nanoporous rocks from primary petrophysical parameters such as surface area, porosity and tortuosity.

\section{INTRODUCTION}

Chalk is a very fine grained natural material with complex pore networks. It is composed predominantly of submicrometer diameter crystals of biogenic calcite that originated as elements in coccoliths, the shields that are created by coccolithophorids, the species of algae that use calcium carbonate to cover their single cell. Extensive chalk deposits were formed during the Cretaceous period (146 to $66 \mathrm{Ma}$ ago), in a number of large sedimentary basins at the edges of continents. Chalk porosity can range from only a few percent to more than $50 \%$ but its permeability is usually very low, in the millidarcy range. The permeability of a porous material determines the rate at which a fluid can be transported through its pore system under external fluid pressure. Porous chalk can serve as drinking water aquifers, sites for $\mathrm{CO}_{2}$ storage and oil or gas reservoirs, so information about their permeability is essential for estimating their economic and technical feasibility.

The Kozeny-Carman equation [1] is one of the simplest ways to estimate permeability, $k$, of a porous material. It combines surface area, A, porosity, $\varphi$, and a material specific constant, $K$ :

$$
k=\frac{\varphi^{3}}{K A^{2}}
$$

The constant, $K$, depends strongly on the tortuosity of the sample, which in turn is a measure of how far the actual pore system deviates from a network of independent cylindrical channels that 
run parallel to the pressure gradient. Tortuosity is a measure of how twisted the pore system is. In rocks, it varies significantly from one rock type to another because different grain shapes, sizes, degrees of compaction and microfractures influence the distribution of the pores in the rock. Thus for some rocks, such as highly compacted chalk, the tortuosity can be quite large. A range of empirical descriptions for the dependence of tortuosity on porosity has been proposed $[2,3]$ and for some simple grain shapes, analytical shape factors are used [4]. More recent attempts even take the fractal dimension of the porous media into account [5]. However, in practice, $K$ is often simply used as a correction factor to fit Eq. 1 to experimental data. As a result, the physical meaning and value of these fitted $K$ parameters depend on the context, i.e. the type of measurement performed, to derive the permeability. For example, measurements of the conductivity of electrical current in response to an external electric field can give different results for $K$ and thus the tortuosity, than measurements of hydraulic conductivity, the fluid flow as a result of an applied pressure gradient. Therefore, it is common to differentiate between hydraulic, electrical, geometric and diffusional tortuosity [6].

Direct experimental measurements of any type of tortuosity are notoriously difficult but can be achieved using for example magnetic resonance of thermal and laser polarized gas [7, 8]. Computerized X-ray tomography now offers the opportunity to image the pore system of a rock directly, with very high resolution, so the geometric tortuosity, i.e. the ratio of the distance connecting two points by a straight line and the shortest distance through the pore system, can be derived from these data using direct measurements or geodesic reconstructions [9]. For the diffusional tortuosity, random walk simulations following the Monte Carlo method [10, 11] can be used while the hydraulic tortuosity is accessible through simulations of flow using the Lattice Boltzmann method $[12,13,14,15]$. 
In this article, we have focussed on the diffusional tortuosity. For sandstones, it has already been shown that random walk simulations in the pore system can produce reliable results for tortuosity [11]. For chalk however, this has not been feasible because of small pore size and the limited resolution that was obtainable using X-ray tomography. Over the past decades, the capabilities of tomography have increased tremendously, with advances in technical equipment and especially from using monochromatic synchrotron radiation. At the highest resolutions available (around 25 $\mathrm{nm}$ voxel size $[16,17])$, it is now possible to image pore systems in chalk.

Alongside the developments of X-ray techniques, computational power has also increased dramatically, which allows us to go further than the random walk approach, by studying the impact of two additional parameters: i) the size of the diffusing particles and ii) their interactions with the pore surface. Particle size is important because in some highly compacted chalk types, the pore throats connecting the individual pores can be so small (less than $100 \mathrm{~nm}[18]$ ) that some high molecular weight oil components are on same length scale as the pores. Consequently, the dynamics of the molecules in the pores becomes size dependent, which leads to a size dependent tortuosity. An additional aspect is that attractive interactions between the oil/water/gas molecules and the pore surface are known to decrease the fluid transport velocity. Thus, both the particle size and its interaction with the pore surface impact the fluid transport properties of the pore network, leading to an "effective" tortuosity.

The effective diffusional tortuosity through a pore network can be determined by examining the movement of particles over time as a function of their size and the energy of interaction with the pore surfaces in comparison with unrestricted diffusion. Some attempts have been made to include sorption [19] in random walk simulations but these have mostly been restricted to one dimensional pore channels [20]. In this paper, we use a full three dimensional model for two 
different types of chalk that were sampled from cores drilled in the Hod Formation in the North Sea Basin, to derive the effective diffusional tortuosity. The pore structures of these samples have been determined using X-ray tomography and imported into a mesoscale dissipative particle dynamics (DPD) simulation [21] using spherical mobile particles.

\section{METHODS AND MATERIALS}

As proposed by Nakashima and Watanabe [11], simulations of particles diffusing in a 3D pore system, such as can be constructed from tomography data, can be used to estimate the diffusional tortuosity in a sample. Together with the porosity and surface area, both easily accessible from the tomograms, the derived tortuosity leads to the permeability via a modified Kozeny-Carman equation:

$$
k \approx \frac{\varphi \cdot \frac{D_{\text {sample }}}{D_{\text {free }}}}{(1-\varphi)^{2} \cdot\left(\frac{S}{V}\right)^{2}}
$$

where $\varphi$ represents the porosity and $S / V$, the surface to volume ratio. The diffusional tortuosity enters Eq. 2 as the inverse of the ratio of two diffusion constants $\left(D_{\text {sample }} / D_{\text {free }}\right)^{-1}$, where $D_{\text {free }}$ and $D_{\text {sample }}$ characterize diffusion in the bulk and through the pore system. Accordingly, $\left(D_{\text {sample }} / D_{\text {free }}\right)$ in Eq. 2 is proportional to $K^{-1}$ in Eq. 1 .

A diffusion constant is defined as the infinite time limit of the time derivate of the mean square displacement (MSD):

$$
D(t)=\frac{1}{6} \cdot \lim _{t \rightarrow \infty} \frac{d\left\langle r(t)^{2}\right\rangle}{d t}
$$


In practise, this means that the diffusion constant would be calculated from the time derivative of the MSD where it becomes constant, i.e. the MSD becomes a linear function of time. Instead of using a voxel sized random walker on a lattice as in [11], we used actual particles in a DPD simulation, i.e. not on lattice. These particles could be varied in size and in their ability to interact with the pore surfaces. Thus the tortuosity derived by calculating $\left(\mathrm{D}_{\text {sample }} / \mathrm{D}_{\text {free }}\right)$ is an effective diffusional tortuosity that includes the size and interaction effects.

X-RAY TOMOGRAPHY. The porosity and surface area of porous rocks in general, but especially for chalk, vary considerably depending on the level of consolidation. For this work, we worked with two Hod chalk (HC) samples taken from drill cores from the North Sea Basin, namely: i) medium porosity chalk (MPC), HC \#15 and ii) low porosity chalk (LPC), HC \#70 [18]). Their porosity differences result from their diagenetic history so one is more consolidated than the other. Tomography data for the less consolidated, MPC, were recorded at the European Synchrotron Research Facility (ESRF) in Grenoble, France, using the setup at the nanotomography beamline ID22 [16]. This setup makes it possible to take images at a range of resolutions, in this case varying in voxel size from 320 to $25 \mathrm{~nm}$. However, because the features of the pore system are at about the same scale as the best obtainable resolution, namely voxel size $=25 \mathrm{~nm}$, we use only this data set here. Data for the highly compacted LPC were recorded at the Swiss Light Source (SLS) [17] in Villigen, Switzerland, using the ptychographic X-ray tomography method [22] at approximately the same voxel size $(21.5 \mathrm{~nm})$.

To segment the tomograms, we used the approach reported by Müter and colleagues [23], which uses a filtering step followed by traditional Otsu thresholding. In the case of the MPC, the data contained ring artefacts which we removed using the method described by Jha et al. [24]. Some of the primary petrophysical parameters, i.e. porosity and surface area, which were derived from 
tomograms for these samples, have already been published elsewhere [18]. In Figure 1, 3D subvolumes of the reconstructed data for both samples are shown before and after segmentation. The segmented volumes are represented as both the material phase and the pore network. It is obvious that the loosely consolidated MPC has a large, well connected pore system, whereas the more compact LPC has only a few larger pores that are connected by narrow fractures that are $\sim 100$ nm wide.

The porosity and specific surface area determined from the tomogram for MPC are $25.7 \%$ and $1.58 \mathrm{~m}^{2} / \mathrm{g}$ and for LPC, they are $5.2 \%$ and $0.50 \mathrm{~m}^{2} / \mathrm{g}$ [18]. There is a broad distribution of pore sizes because the grains in chalk are generally quite irregular. For LPC, average pore diameter is $\sim 9.5$ voxels $(\sim 240 \mathrm{~nm})$ with a standard deviation of 5.1 voxels $(\sim 128 \mathrm{~nm})$. For MPC, pores are on average larger, $\sim 12.7$ voxels $(\sim 317.5 \mathrm{~nm})$ with a standard deviation of 4.1 voxels $(\sim 103 \mathrm{~nm})$. Although the porosity for LPC is quite low, the bulk of the pore system is connected, leaving only $11 \%$ of the total porosity inaccessible, i.e. $0.6 \%$ of the whole volume is inaccessible pore space. In the last preparation step for the DPD simulation, the inaccessible pores were removed to avoid model particles that would initially be trapped inside those pores at the start of the simulation, which would bias the mean square displacement estimation. 

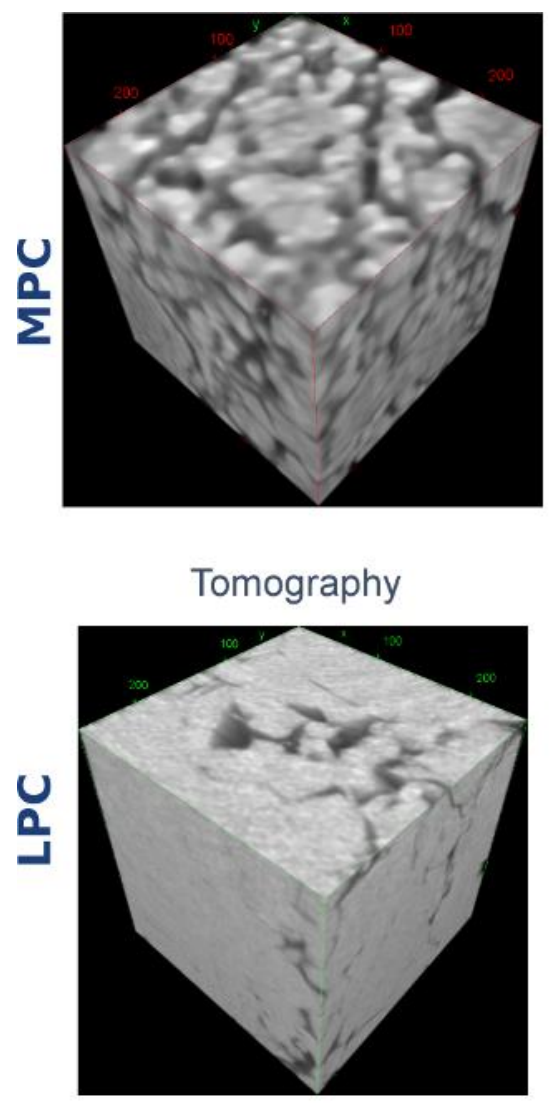
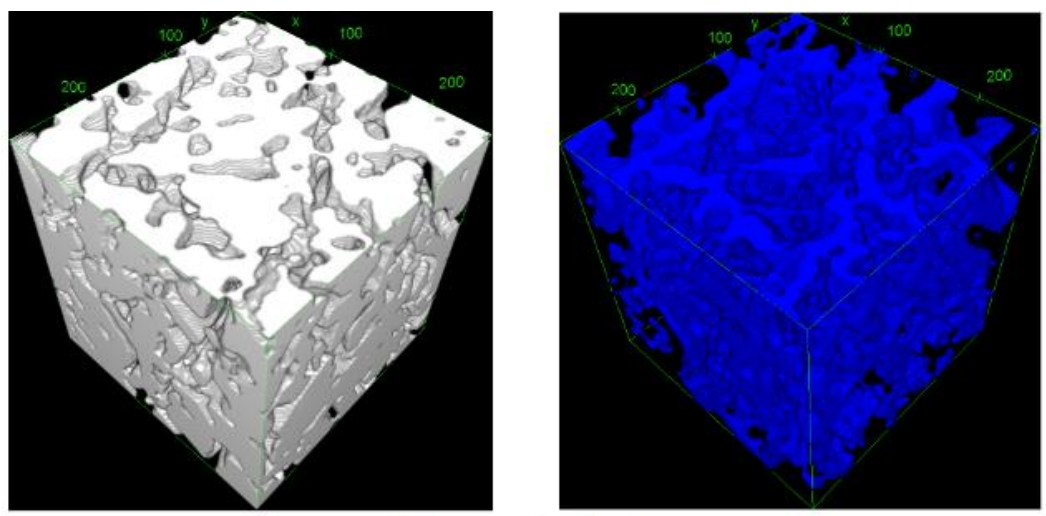

Segmented into

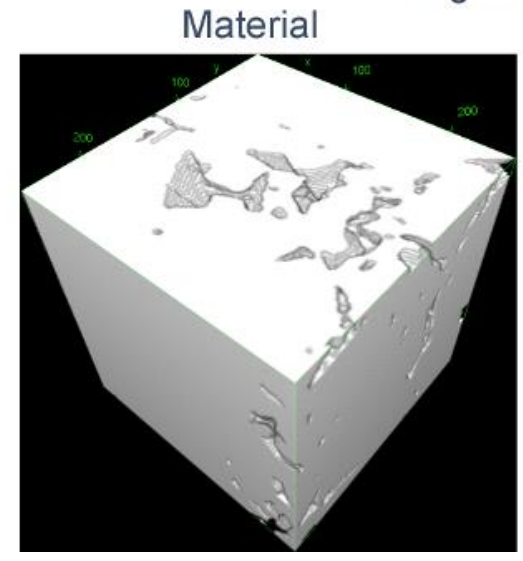

Pores

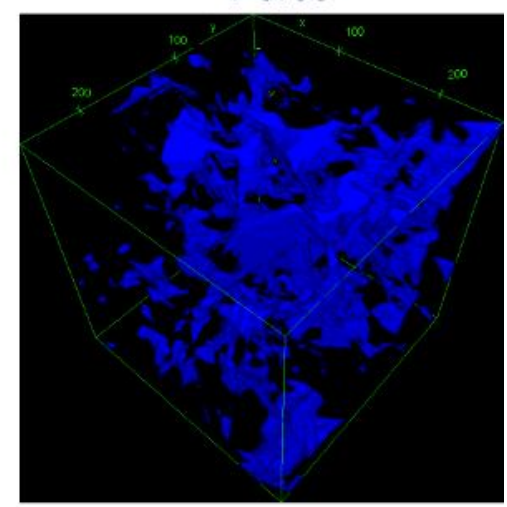

Figure 1: Subvolume cubes of the reconstructed tomography data for both samples (edge length: $\sim 6.4 \mu \mathrm{m}$ ) and segmented to represent the material phase and pore network (unconnected pores have not been removed yet). The DPD simulation box (Fig. 2) is a subvolume cube that is $4 \mu \mathrm{m}$ on a side, extracted from the data shown here.

\section{DISSIPATIVE PARTICLE DYNAMICS (DPD) SIMULATIONS}

We used the DPD method to simulate particles diffusing through the pore system. This technique has previously been applied successfully to study adsorption and aggregation of coarse grained molecules in simple geometries such as cylindrical pores [25] and nanofibres [26, 27, 28]. For these simple geometries, the interaction between the confining surface and the particles, i.e. the force field, can usually be described by an analytical function, such as the Lennard-Jones (LJ) 
potential. Because of the strongly irregular surface of the chalk pore system (Fig. 1), we must approximate it by simpler geometric elements. One could in principle use a polygonal surface representation [29] but it is challenging to avoid discontinuities in the force field at the edges and corners of nonparallel polygons. Instead, we place an immobile spherical particle with diameter that is 1.2 times the voxel edge length (i.e. $\sigma_{\text {surf }}=1.2 * 25 \mathrm{~nm}$ ) at the centre of each surface voxel. A surface voxel is defined as any material voxel that shares a face with a void (pore) voxel. This leads to a "bumpy" approximation of the surface. Therefore, another surface particle is positioned between any two existing particle positions by interpolating in all three dimensions. For the size of our simulation box $(4 \mu \mathrm{m})^{3}$ (Table 1) this leads to $10^{5}-10^{6}$ surface particles. To keep the computational efforts manageable, the surface particles were kept immobile and interactions between them were not permitted. Accordingly, only interactions between surface and fluid particles had to be evaluated.

Table 1: Parameters and settings used in the DPD simulations

\begin{tabular}{|l|l|l|}
\hline$\sigma_{\text {fluid }}=0.1-3.0$ & $\varepsilon=0.1-0.25$ & $\Delta \mathrm{t}=0.0005-0.005$ \\
\hline$\sigma_{\text {surf }}=1.2$ & $\mathrm{t}_{\text {equi }}=0.4-4 * 10^{7}$ steps & Box-dim $=(160)^{3}$ \\
\hline temp $=0.5 \varepsilon / \mathrm{k}_{\mathrm{B}}$ & $\mathrm{t}_{\text {prod }}=0.1-1 * 10^{7}$ steps & $\mathrm{N}_{\text {fluid }}=20-1200$ \\
\hline
\end{tabular}

The particles representing the molecular fluid in this simulation were spherical and they were randomly inserted into the pore network at the start of the simulation. We introduced two types of particles: N1 had no attractive interaction with pore surfaces, while $\mathrm{H} 1$ particles were attracted by them. The fluid/fluid interparticle interactions, i.e. the $\mathrm{H} 1 / \mathrm{H} 1$ and the $\mathrm{N} 1 / \mathrm{N} 1$ interactions were purely repulsive to avoid any condensation effects that would add another level of complexity. 
The diameter of both types of particles, $\sigma_{\text {fluid, }}$ was also defined relative to the voxel edge length $(25 \mathrm{~nm})$. To simulate molecules of different sizes, we varied $\sigma_{\text {fluid }}$ between 0.1 and 3.0

(corresponding to $2.5-75 \mathrm{~nm}$ ). In our model, the distance at which a fluid particle and a surface particle touch is given by the Lorentz mixing rule, i.e. $\sigma_{\mathrm{fs}}=\left(\sigma_{\text {fluid }}+\sigma_{\text {surf }}\right) / 2$. This ensures that the force fields from all surface particles overlap locally in a way that fluid particles cannot pass through the fluid-solid interface. The H1 particles interact with the surface via the full LJpotential, thus allowing adsorption of the particles on the pore surfaces:

$V_{L J}=4 \varepsilon_{\mathrm{H}}\left\{\left(\frac{\sigma}{r}\right)^{12}-\left(\frac{\sigma}{r}\right)^{6}\right\}$

All other interactions are soft repulsive and represented by the LJ-potential truncated at $\mathrm{rmin}=$ $2^{1 / 6} \sigma$.

For fluid/fluid interactions, the interaction strength, $\varepsilon_{\mathrm{H}}$, was set to 1 . To simulate different adsorption energies, $\varepsilon_{\mathrm{H}}$ was multiplied by a factor, $0.1<\varepsilon<0.25$, for fluid/wall interactions. The temperature was set to $0.5^{*} \varepsilon_{\mathrm{H}}$, leading to an average kinetic energy per particles of $3 / 2 * \mathrm{~T}=$ $3 / 4 * \varepsilon_{\mathrm{H}}$. Thus, the interaction energy per interacting fluid/surface particle pair was always less than the average kinetic energy of the fluid particle. This ensured that the particles were not adsorbed so strongly to the surface that they could not move at all.

Because of the irregular shape of the pore system, the simulation box cannot be periodic. To ensure that particles could not leave the simulation box, we introduced walls with a soft repulsive potential on all six sides. This required an adjustment for the calculation of the mean square displacement (MSD) because it introduced an artificial upper boundary to the MSD. Moreover, particles that have been reflected by the outer walls of the simulations box would tend to diffuse 
back in the direction they came from, thus decreasing the MSD. Accordingly, we record whenever a particle comes into contact with the outer walls and take this particle out of the calculation of the MSD. It is, however, still present in the simulation and can interact with other wall and fluid particles. The number of "active" particles with respect to the MSD calculation decreases with time so statistical deviations from the mean value increase. We stopped the evaluation of the MSD curves when less than $\mathrm{e}^{-1}$ of the initial number of particles were still active. Figure 2 shows the setup for the DPD simulation for the LPC along two different axes.
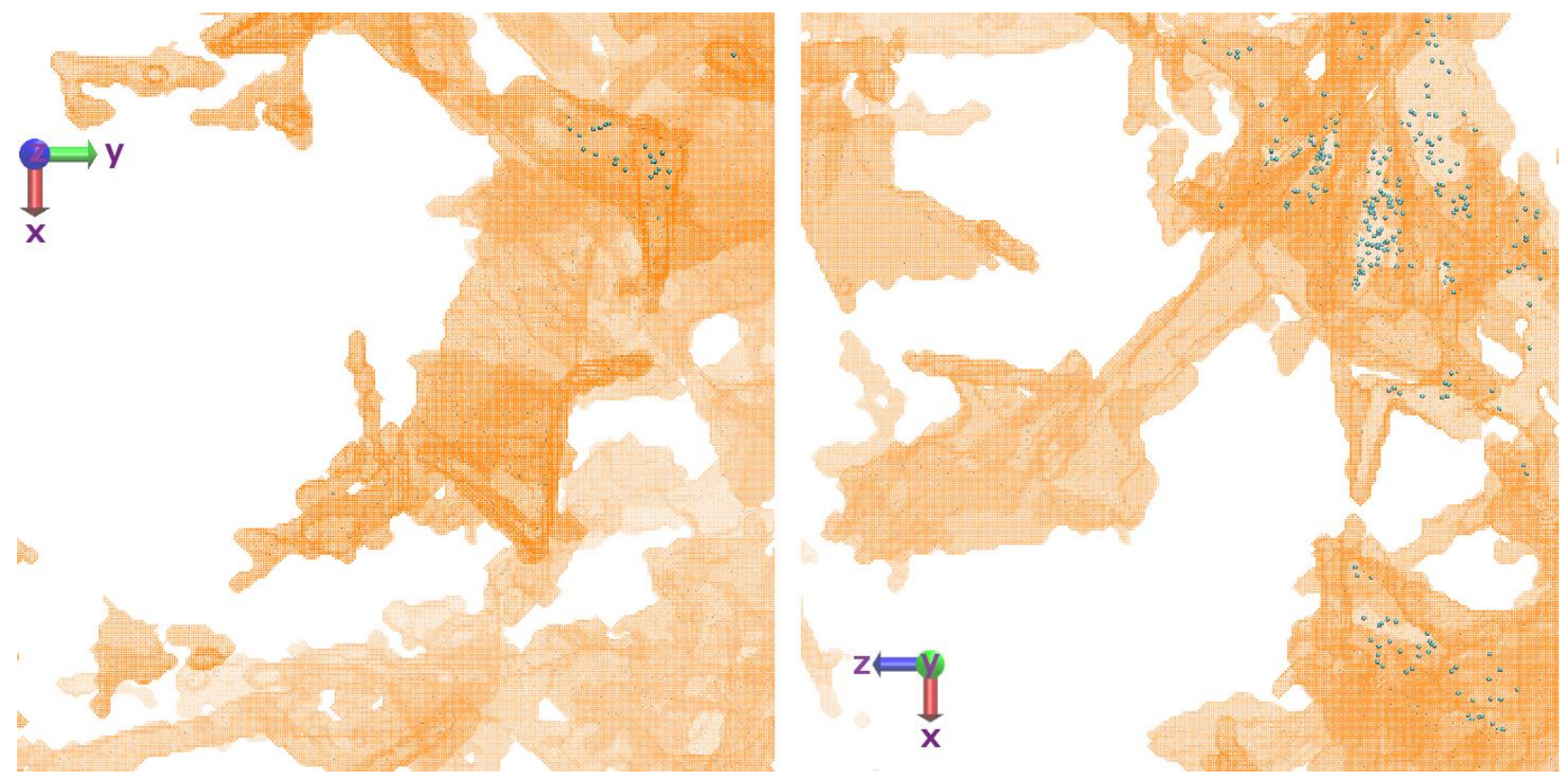

Figure 2: Cross sections of the starting conditions for the DPD simulation on LPC along the $\boldsymbol{z}$ axis (left) and $\boldsymbol{y}$-axis (right) with surface particles (orange) and fluid particles (cyan). Only the fluid particles near the entrance to the pore system can be seen. Note that the bulk of the chalk (solid material and unconnected pores) does not play any role in the simulation and is therefore transparent. 
In Eq. 2, the MSD of the fluid particles within the pores is compared with that of free diffusion. Accordingly, we also simulated N1 particles in an empty box and recorded the MSD after an initial equilibration phase. To retrieve meaningful results for the MSD, it is essential to keep the temperature constant. The DPD method regulates the temperature with a dissipative term in the particle interaction potentials. This requires an alteration of the time step length to suit the particle diameter, i.e. shorter time steps for larger particles, as described in the original article about DPD [21]. To reach the same total simulation time, the number of time steps for equilibration and data production must be adjusted as well. The individual simulations were performed in the canonical ensemble (NVT), i.e. in addition to the temperature, the volume of the simulation box and the number of particles were both kept constant.

Increasing the diameter of the fluid particles means that more of the pore volume could be taken up by the fluid particles, to the point where the system would be essentially full and the particles would have a very short mean free path. This, however, is not an effect of the tortuosity of the sample. Instead of using the same number of particles for all simulations, we adjusted the particle number to keep the volume fraction of fluid particles constant. Performing the simulations at very low density was then favourable but a larger number of particles yielded better statistics. We determined the optimal fluid density by increasing the number of particles for an intermediate particle diameter $\left(\sigma_{\text {fluid }}=0.5\right)$ and examining the MSD. For LPC, the MSD began to decline at $\mathrm{N}=2,500$, corresponding to a fluid volume fraction of $1 \%$ of the pore space. Accordingly, for all simulations, the fluid particle volume in the pores was kept at $<1 \%$. Because of the higher porosity of the MPC sample, the same number of particles was sufficient because they take up less of the pore volume.

\section{RESULTS AND DISCUSSION}


The MSD curves for the N1 particles in the pore system, plotted in Figure 3, show a relatively short initial phase, where MSD increase rapidly followed by a slower, nearly linear increase. The first phase is associated with the initial, unhindered movement of the particles before they interact with the pore surfaces. The pores in MPC are on average larger than in LPC (Fig. 2) so this phase is longer for MPC. The behaviour after the initial phase can be described as subdiffusive because the increase in MSD is, by far, lower than for the curve showing free diffusion. The diffusion process in LPC is even slower.

Increasing the particle size had comparatively little influence on the MSD in MPC. For LPC, there is a clear drop in the MSD, suggesting that for the larger particles (1.5 $\sigma$ corresponds to $16 \%$ of the average pore diameter) the smaller fractures in that sample impede particle movement.
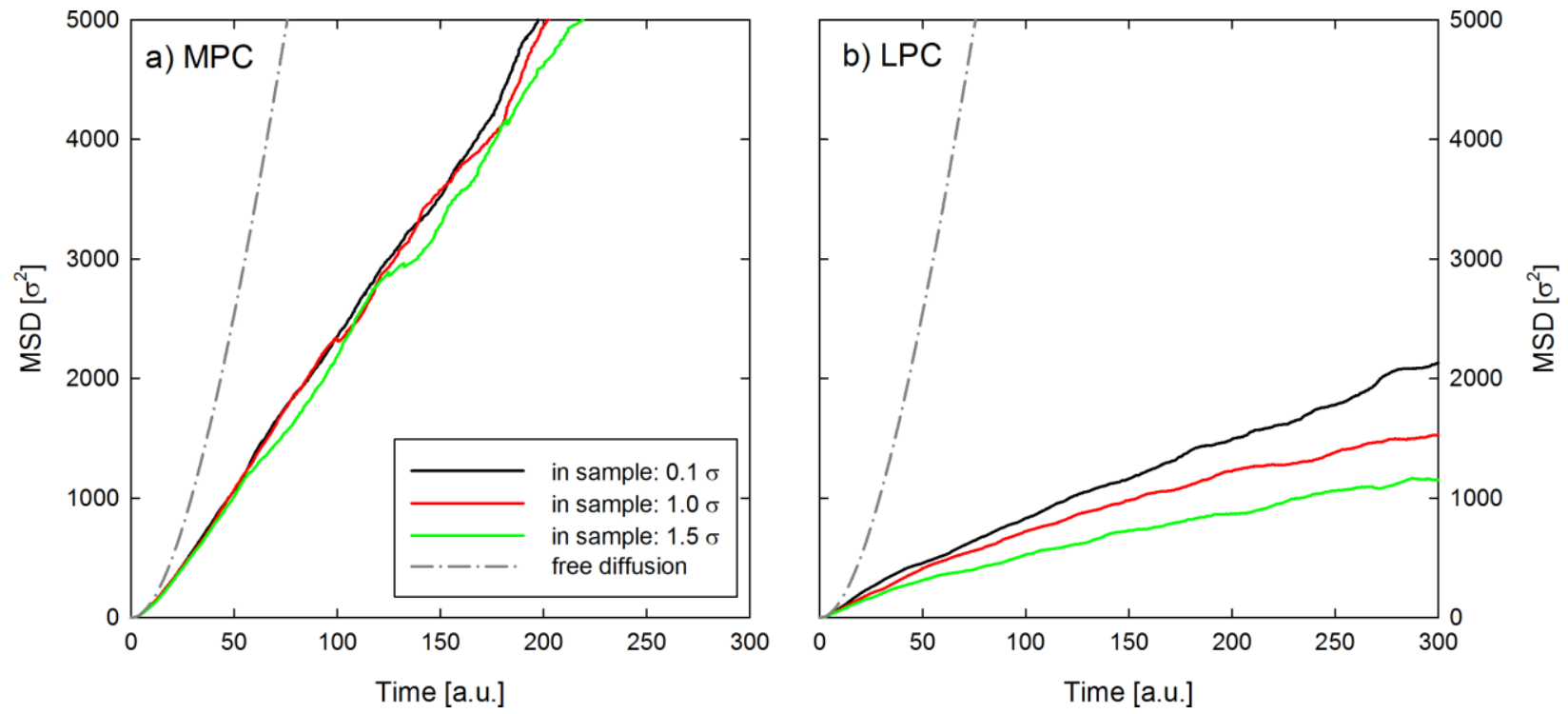

Figure 3: Mean square displacement (MSD) for N1 particles (repulsive interaction only) with different diameters in the pore system of MPC (a) and LPC (b) compared with free diffusion. 
In Figure 4, MSD curves are shown for $\mathrm{H} 1$ particles in both samples at two different interaction strengths. For both samples, the weaker interaction strength of $\varepsilon=0.1$ has a noticeable influence on diffusion and intensifies the effect of larger particle size, i.e. the diffusion of larger particles is impeded even more. Diffusion is severely inhibited at the higher interaction strength, i.e. $\varepsilon=$ 0.25 , in both samples. The strong dependence of the MSD on particle size can be explained by the particle size dependence of the interaction range. For larger particles, there are on average more surface particles within the attractive region of the LJ-potential and accordingly, adsorption is stronger. Assuming homogenous chemical behaviour over the particle, i.e. molecule, this is exactly the same for the real pore surface, where larger fluid molecules cover more surface area and therefore interact more strongly than smaller molecules. The coupling between particle size and interaction strength and its impact on the MSD is quite interesting because the particle diameters in question $(0.1 \sigma$ is equivalent to $2.5 \mathrm{~nm})$ are on the same length scale as the heavier compounds in certain types of crude oil. Depending on the chemical behaviour of the surface, reservoir rocks can be oil wet, water wet or mixed wet. Accordingly, there are oil reservoirs in which at least some of the oil components are strongly attracted to pore surfaces, as in the simulations here. In this case, even for weak interactions with pore walls, the rate of transport of these molecules could be decreased considerably and the production rate would be less than predicted from initial estimates based on Eq. 1. 

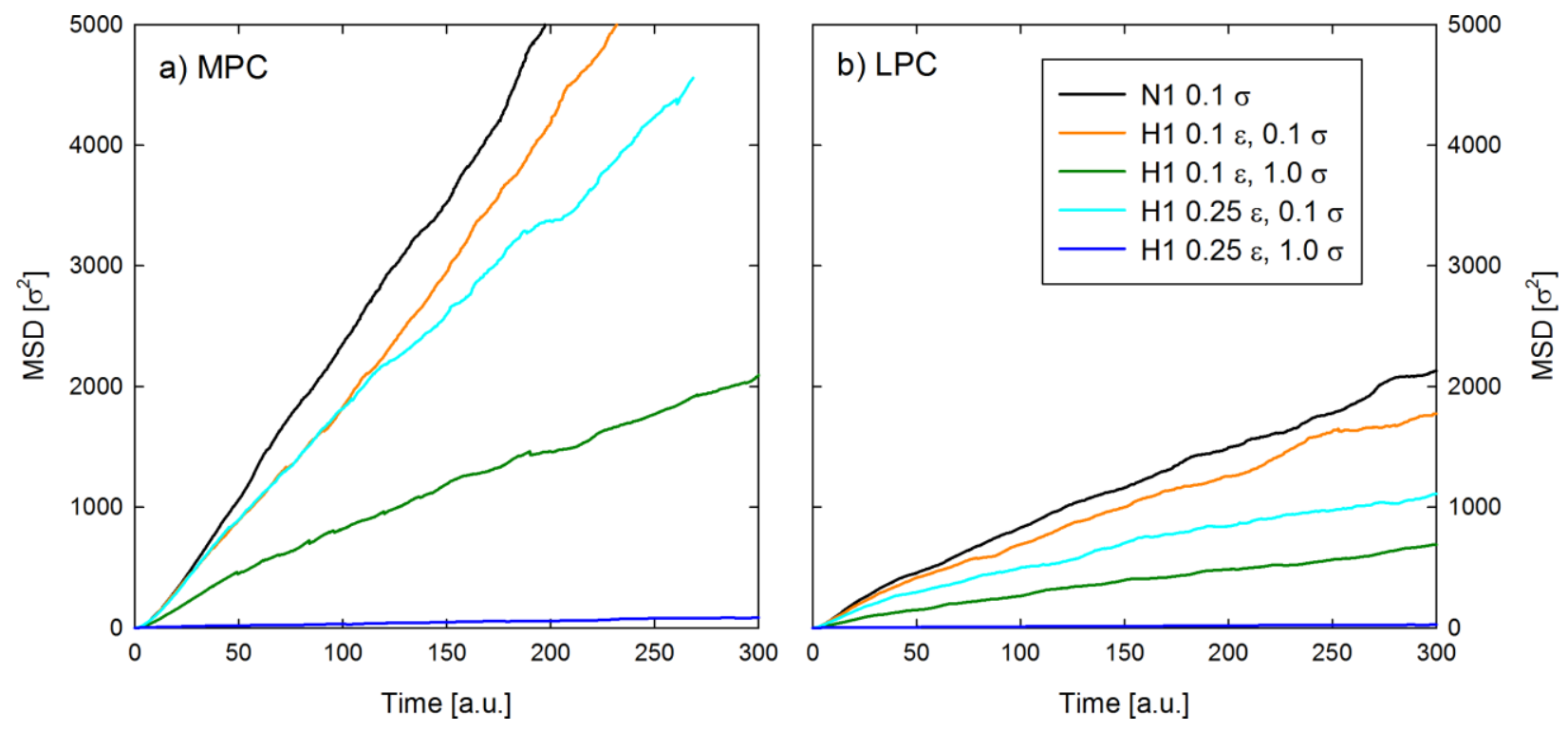

Figure 4: Mean square displacement for $\mathrm{H} 1$ particles (particle-particle interaction is repulsive; particle-surface interaction is attractive) with different diameters in the pore system of MPC (a) and LPC (b) for limited attractive interaction with the surface and stronger interaction in comparison with N1 particles. The colour code indicted in part (b) is used for the entire figure.

To estimate the time derivatives of the MSD curves, we divide the change in MSD between each time step by the time step size. From the resulting curve $\left(d\left\langle r(t)^{2}\right\rangle / d t\right.$ in Eq. 3$)$, the average, i.e. the diffusion constant, is determined from the region where MSD is linear, e.g. from 50 to 300 time units in Figure 4b. Doing so for MPC, LPC and free diffusion leads to the ratio of diffusion constants as a function of particle size and interaction strength (Figure 5). As expected, diffusion in the MPC is much faster than in the LPC. For attractive interactions with the surface $(\mathrm{H} 1$ particles), diffusion decreases rapidly and becomes strongly dependent on particle size. Naturally, stronger interaction $(\varepsilon=0.25)$ leads to stronger adsorption and slower diffusion. For example, at $\varepsilon=0.1$ and $\sigma=0.1,30 \%$ of all particles are adsorbed onto the surface. Setting $\varepsilon$ to 0.25 , with the same particle size, increases the proportion of adsorbed particles to $45 \%$. For 
larger particles, this effect is even more pronounced, to the point where nearly all particles are strongly adsorbed, i.e. at $\sigma=1.0$. Thus, at higher interaction with the surface, particle movement becomes more and more restricted to the surface diffusion, where particles are trapped by heterogeneities.

From the ratio of diffusion constants, we can directly calculate the diffusional tortuosity of the pore network by taking the inverse. Without interaction with the surface, this leads to values of 4 to 10 for MPC and 16 to 50 for LPC. This is far higher than the value reported for sandstone (1.6) by [11] but it is within the limits given by analytical solutions for a selection of grain shapes [4]. Thus, because of the very low porosity in chalk and the complex pore structure, as we see in Figure 1, these high tortuosity values are realistic and demonstrate convincingly that tortuosity has to be estimated carefully if it is to be used to predict permeability in rocks with unknown or complicated structure.
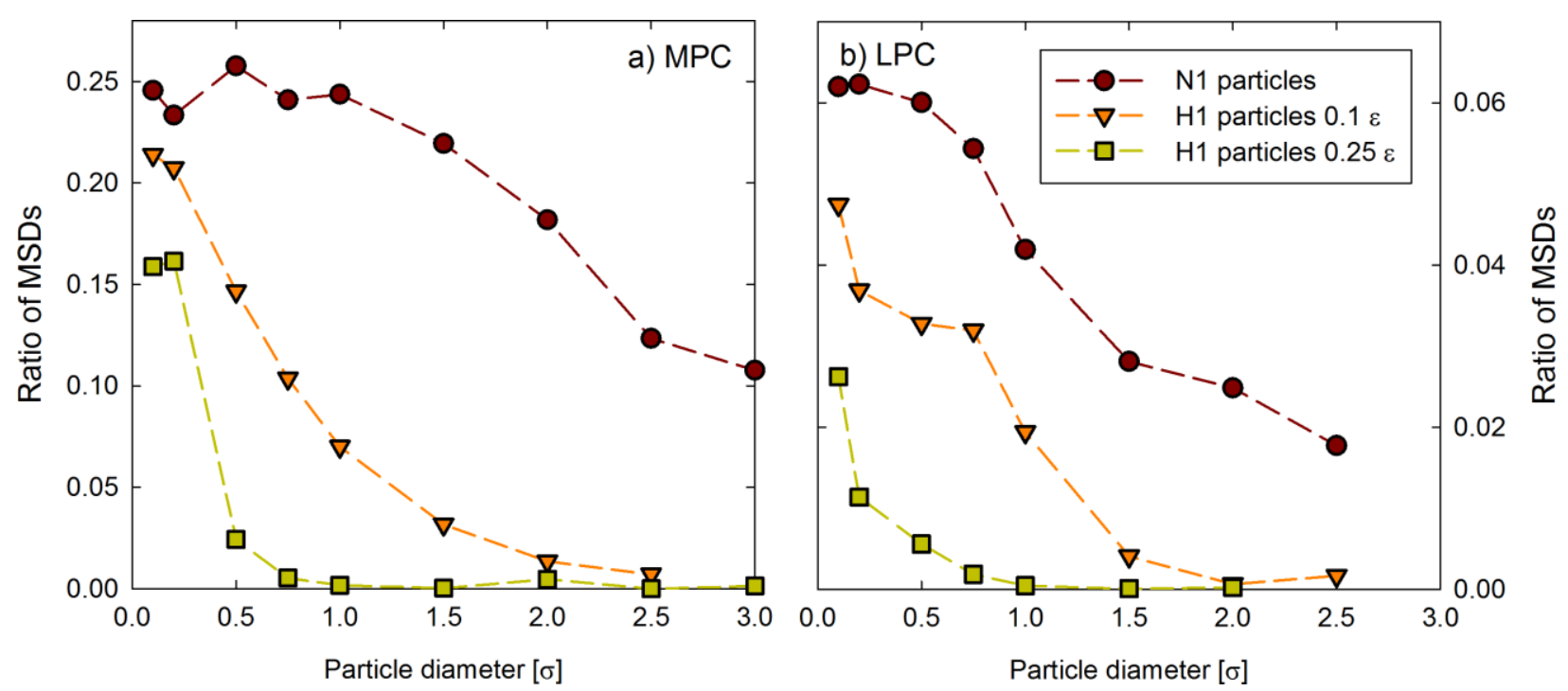
Figure 5: Average ratio of mean square displacement (free diffusion =1) for MPC (a) and LPC (b) calculated from the MSDs, for different particle sizes and interaction strengths shown in Figures 3 and 4.

\section{CONCLUSIONS}

We have studied the diffusion of particles of variable size and strength of interaction with surfaces in the highly irregular pore system of chalk. Exploring particle behaviour in "real" pore geometry, derived from X-ray nanotomography, is a clear step forward, compared with what has been done until now, namely common studies on model systems that have high degrees of symmetry and simple pore geometry. In comparison with earlier studies that used comparable tomography data, for example from sandstone, and with a lattice based random walk, our approach makes several advances. The resolution is a factor of at least 100 times higher, our modelling incorporates arbitrary sized particles and it can be used to investigate adsorption and aggregation phenomena, as well.

The relative diffusion constants that we have derived here are related directly to experimentally accessible and widely relevant properties. In particular, the inverse of the ratio of diffusion constants gives the diffusive tortuosity of the sample. We found that diffusion is impeded when particle size is larger, especially in the highly compacted LPC sample, which means that the tortuosity effectively increases. Adsorption to pore surfaces, simulated here by introducing an attractive fluid/solid interaction, dramatically intensifies this effect. In some cases, diffusion is almost completely subdued, which demonstrates that both factors, particle size and adhesion properties, have an impact on diffusion, apparent tortuosity and permeability. It is likely that our results will also find application in the private sector (e.g. oil production or contaminant 
remediation) because our approach adds a new level of understanding to fluid flow and transport, particularly when the fluid contains particles or large molecules that have a radius of gyration that approaches the pore throat radius. In oil production and groundwater pollution, some of the heavier organic compounds are in this size range. An important advantage of this work, where for example, the properties of fluid and solid, and their degree of interaction, are known, is that our approach can be applied to derive a more realistic estimate for the tortuosity of the pore system and accordingly, the permeability of the rock.

\section{AUTHOR INFORMATION}

\section{Corresponding Author}

*D. Müter, Nano Science Center, Dept. of Chemistry, University of Copenhagen, Universitetsparken 5, 2100 Copenhagen Ø, Denmark, mueter@nano.ku.dk

\section{Author Contributions}

The manuscript was written through contributions of all authors. All authors have given approval to the final version of the manuscript.

\section{Funding Sources}

Funding was provided by the Danish Advanced Technology Foundation and Maersk Oil and Gas A/S through the P3 project and the Danish Council for Independent Research through DANSCATT. DM acknowledges funding from the People Programme (Marie Curie Actions) of the European Commission Seventh Framework, FP7/2007-2013/ under REA Grant Agreement $n^{\circ} 297921$.

\section{ACKNOWLEDGMENT}


We thank F. Engstrøm for providing the samples, R. Feidenhans'l for scientific advice and H. Suhonen for help with the data collection and reconstruction of the tomograms. Beamtime was provided by the ESRF (European Synchrotron Research Facility) and the SLS (Swiss Light Source).

\section{REFERENCES}

[1] J. Kozeny, Sitzungsber. Akad. Wiss., Wien, 136(2a): 271 (1927)

[2] L. Shen and Z. Chen, Chem. Eng. Sci. 62, 3748-3755 (2007)

[3] B. P. Boudreau, Geochim. Cosmochim. A. 60, 3139-3142 (1996)

[4] P. Xu and B. Yu, Adv. Water Resourc. 31, 74-81 (2008)

[5] N. Epstein, Chem. Eng. Sci. 44, 777-779 (1989)

[6] M. B. Clennell, Geol. Soc. Spec. Publ. 122, 299-344 (1997)

[7] R. P. Wang, T. Pavlin, M. S. Rosen, R. W. Mair, D. G. Cory and R. 1. Wandsworth, Magnetic Resonance Imaging 23, 329-331 (2005)

[8] R. Wang, R. W. Mair, M. S. Rosen, D. G. Cory and R. L. Wandsworth, Phys. Rev. E 70, $026312(2004)$

[9] C. J. Gommes, A.-J. Bons, S. Blacher, J. H. Dunsmuir and A. H. Tsou, AIChE Journal 55, 2000-2012 (2009)

[10] P. Epicoco, B. Coasne, A. Gioia, P. Papet, I. Cabodi and M. Gaubil, Acta Materialia 61, 5018-5025 (2013)

[11] Y. Nakashima and Y. Watanabe, Water Resources Research 38, 1272 (2002)

[12] M. Matyka, A. Khalili and Z. Koza, Phys. Rev. E 78, 026306 (2008)

[13] A. Duda, Z. Koza and M. Maytka, Phys. Rev. E 84, 036319 (2011)

[14] A. Ghassemi and A. Pak, Int. J. Numer. Anal. Meth. Geomech. 35, 886-901 (2011) 
[15] S. Kynde, F. Engstrøm, T. H. Jensen, R. Feidenhans'l and S. L. S. Stipp, in preparation (2014)

[16] T. Weitkamp, C. Raven and A. Snigirev, Developments In X-ray Tomography II, edited by U. Bonse, Proceedings SPIE 3772, 311 (1999)

[17] M. Holler, A. Diaz, M. Guizar-Sicairos, P. Karvinen, E. Färm, E. Härkönen, M. Ritala, A. Menzel, J. Raabe and O. Bunk, Sci. Rep. 4, 3857 (2014)

[18] D. Müter, H. O. Sørensen, D. Jha, R. Harti, K. N. Dalby, H. Suhonen, R. Feidenhans'l, F. Engstrøm and S. L. S. Stipp, Appl. Phys. Lett. 105, 043108 (2014)

[19] Y. Seida, H. Takase, H. Takahashi and Y. Niibori, Materials Research Society Symposium Proceedings 1124, 543-548 (2009)

[20] S. Chatterjee and G.M. Schütz, Micropor. Mesopor. Mater. 125, 143-148 (2009)

[21] R. D. Groot and P. B. Warren, J. Chem. Phys. 107, 4423 (1997)

[22] H. N. Chapman, Nature 467, 409 (2010)

[23] D. Müter, S. Pedersen, H. O. Sørensen, R. Feidenhans'l and S. L. S. Stipp, Computers \& Geosciences 49, 131 (2012)

[24] D. Jha, H. O. Sørensen, S. Dobberschütz, R. Feidenhans'l and S. L. S. Stipp, Appl. Phys. Lett. 105, 143107 (2014)

[25] D. Müter, M. A. Widmann and H. Bock, J. Phys. Chem. Lett. 4, 2153 (2013)

[26] D. Müter, P. Angelikopoulos, H. Bock, J. Phys. Chem. B 116, 14869 (2012)

[27] D. Müter and H. Bock, J. Phys. Chem. B 117, 5585 (2013)

[28] D. Müter and H. Bock, Phys. Rev. Lett. (2014)

[29] W. E. Lorensen and H. E. Cline, SIGGRAPH '87 Proceedings of the 14th Annual Conference on Computer Graphics and Interactive Techniques, 163 (1987) 


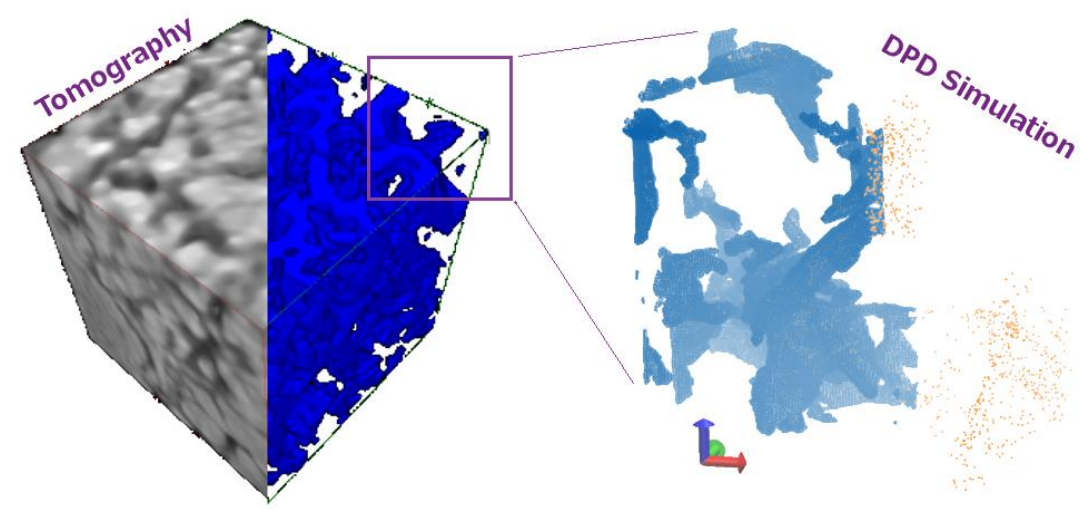

Table of Contents Graphic 